\title{
Distribution des espèces de primates au Bénin et ethnozoologie
}

\author{
G. NOBIME * , O.G. GAOUÉ et B. SINSIN \\ Laboratoire d'Ecologie Appliquée, Faculté des Sciences Agronomiques, Université d'Abomey-Calavi, 01 BP \\ 526 Cotonou, Bénin. \\ *Auteur correspondant, E-mail: gnobime@yahoo.fr
}

\section{RESUME}

La République du Bénin appartient au Sillon dahoméen et abrite des espèces de primate. Face à la dégradation rapide du couvert végétal avec son cortège de flore et de faune, il urge de mieux apprécier la situation afin d'une meilleure conservation du potentiel naturel disponible actuellement. La présente étude a pour but de faire le point de l'état de la diversité des primates au Bénin. Elle est le fruit de 1253 jours de prospections, d'enquêtes et d'observations dans les grands écosystèmes du pays, de façon sporadique, entre 1996 à 2006, le tout soutenu par la documentation. Le Bénin possède 10 espèces de primates confirmées et une autre espèce le mangabey qui reste à confirmer. Ces différents taxa de primates appartiennent à des écosystèmes variés. Certaines espèces sont communes comme la mone Cercopithecus mona, le babouin Papio anubis, le tantale Chlorocebus t. tantalus, par contre d'autres sont rares et inféodées à des milieux particuliers comme le cercopithèque à ventre rouge Cercopithecus e. erythrogaster qui est une espèce endémique du pays. Les populations locales utilisent quelques organes de certaines espèces de primates pour guérir des maladies et/ou réaliser des procédés médico-magiques.

(C) 2008 International Formulae Group. All rights reserved.

Mots clés : Diversité primates, procédés médico-magiques, Bénin, Sillon dahoméen.

\section{INTRODUCTION}

Le Bénin appartient au Sillon dahoméen, une frontière entre les blocs forestiers guinéens à l'ouest et congolais à l'est, ce sillon qui n'est que le produit des interactions topographiques, océanographiques et climatiques (Jenik, 1994 ; Natta, 2003). Ce sillon est mis en place à la dernière transgression marine (Salzmann \& Hoelzmann, 2005). Selon Le Gall et al. (2002), il n'est pas une zone tampon vide de toute espèce forestière. Depuis les travaux de Sayer \& Green (1984) relatifs à la faune mammalienne de la république du Bénin, il est apparu que le potentiel du pays en mammifères n'est pas négligeable. Très peu de documents (Kafichoni, 1987; Kassa, 2001 ; Nobimè, 2002; Nobimè et Sinsin, 2003 ; Djossou, 2003 ; Campbell et al., 2008) ont abordé la distribution des espèces de primates abritées par cette partie du Sillon dahoméen. Le présent travail fait le point sur les primates qui existent selon les grandes zones climatiques du pays, les différents écosystèmes leur servant d'habitats, et sur quelques aspects ethnozoologiques. Ceci permettra de donner une idée sur la diversité des primates du pays et quelques-unes de leurs utilisations par les populations locales.

\section{MATERIEL ET METHODES \\ Milieu d'étude}

L'étude s'est déroulée en République du Bénin $\left(112.622 \mathrm{~km}^{2}\right)$ située dans la zone intertropicale de l'Afrique, entre $6^{\circ}$ et $12^{\circ} 50 \mathrm{~N}$ et $1^{\circ}$ et $3^{\circ} 40 \mathrm{E}$. Le pays comporte trois zones climatiques : la zone guinéo-congolaise située entre $6^{\circ} 25$ et $7^{\circ} 30 \mathrm{~N}$, la zone soudanoguinéenne située entre $7^{\circ} 30$ et $9^{\circ} 30 \mathrm{~N}$ et la zone soudanienne située entre $9^{\circ} 30$ et $12^{\circ} \mathrm{N}$.

Le régime pluviométrique est bimodal dans la zone guinéo-congolaise (avril-juin et septembre-novembre) avec une pluviométrie moyenne de $1200 \mathrm{~mm}$ par an; au-delà de $8^{\circ} \mathrm{N}$ et au fur et à mesure que l'on se déplace vers le nord, le régime pluviométrique devient unimodal (mai-octobre) et la pluviométrie moyenne annuelle est globalement supérieure à $900 \mathrm{~mm}$. 
La zone guinéo-congolaise est le domaine des sols ferrallitiques, profonds et peu fertiles (700.000 ha); les sols alluviaux et vertisols (360.000 ha) sont localisés dans les vallées des fleuves Mono, Niger, Couffo et Ouémé, et dans la dépression de la Lama. Ces sols sont riches en argile, en humus et en éléments minéraux. La végétation dans toutes les zones climatiques du Bénin est fortement dégradée par l'homme, surtout dans sa partie méridionale où la densité de la population humaine est élevée. Dans cette région qui correspond à la zone guinéo-congolaise, la végétation est fortement marquée par les activités agricoles. Elle est constituée de jachères et de quelques îlots forestiers de moins de 5 ha. La végétation originelle est constituée de forêts denses semi-décidues et des savanes guinéennes.

Dans le soudanien et le soudanoguinéen, on trouve les sols minéraux peu évolués $(1.500 .000$ ha) et peu fertiles, localisés dans l'Atacora. Les sols ferrugineux sur socle cristallin (8.600. 000 ha), de fertilité variable, sont localisés au centre et au nord. Concernant la végétation, la zone de transition soudano-guinéenne prolongeant la zone à affinités guinéennes, est le domaine des mosaïques de forêts claires, avec éventuellement des forêts denses sèches, parsemées de savanes arborées et arbustives et traversées par des galeries forestières. La zone soudanienne, quant à elle, est constituée de savanes, de pseudo-steppes, de galeries forestières avec des arbres de plus petite taille recouvrant faiblement le sol. La forêt de Pénessoulou située en zone soudanienne constitue l'extrémité nord-est d'une longue ramification du faciès sec du massif forestier occidental de la région guinéenne.

Dans l'ensemble des localités, les principales activités sont l'agriculture, l'élevage extensif et l'exploitation forestière anarchique des forêts claires et forêts galeries.

\section{Méthodologie} surtout :

Les données de la présente étude sont

1) des informations recueillies dans les différentes localités auprès des chasseurs.

Lors de nos différentes missions sur le terrain, des prospections et des enquêtes sont faites en directions des chasseurs et autres personnes connaissant la faune. Ainsi grâce à la mémoire des chasseurs et de planches de photos de primates nous avons obtenu des informations qui nous ont servis à la reconstitution de la faune dans les différentes localités. Les informations sur l'ethnozoologie ont été collectées dans plusieurs localités du Bénin du Sud au Nord.

2) la revue bibliographique à travers les publications scientifiques et autres documents.

Les différentes bibliothèques de l'Université d'Abomey-Calavi au Bénin nous ont permis de noter les différentes espèces de faune rencontrées dans les localités d'étude. Ces informations ont été confirmées par des observations directes et autres enquêtes pour la précision de ces données. Beaucoup d'autres publications scientifiques ont fait le point de la situation de la faune au Bénin.

3) des observations personnelles sur le terrain réalisées de mai 1996 à décembre 2006.

Au total, 1253 jours, de façon sporadique, de prospections, d'enquêtes et d'observations dans les grands écosystèmes du pays, ont permis de collecter des données.

\section{RESULTATS \\ Diversité des primates et de leurs habitats}

La distribution des espèces de primates au Bénin recoupe la fragmentation des massifs forestiers du Sillon dahoméen. Les stations explorées dans chacune de ces zones climatiques sont indiquées dans les figures 1 et 2 .

Avec le tableau 1, nous remarquons que la zone guinéo-congolaise abrite, à elle seule, huit (8) taxa de primates à savoir trois espèces nocturnes, le Potto de Bosman Perodicticus potto, les galagos Galago senegalensis, Galago demidovii et cinq espèces diurnes la mone Cercopithecus mona, le cercopithèque à ventre rouge Cercopithecus e. erythrogaster, le tantale Chlorocebus $t$. tantalus, le colobe de Geoffroy Colobus vellerosus et le colobe de Van Beneden Procolobus verus. Le mangabey Cercocebus torquatus, dont nous avons observé des trophées auprès des chasseurs riverains de la forêt classée de la Lama et de la forêt marécageuse de Lokoli, portera à 9 le nombre de taxa dans cette zone quand sa présence sera confirmée. 


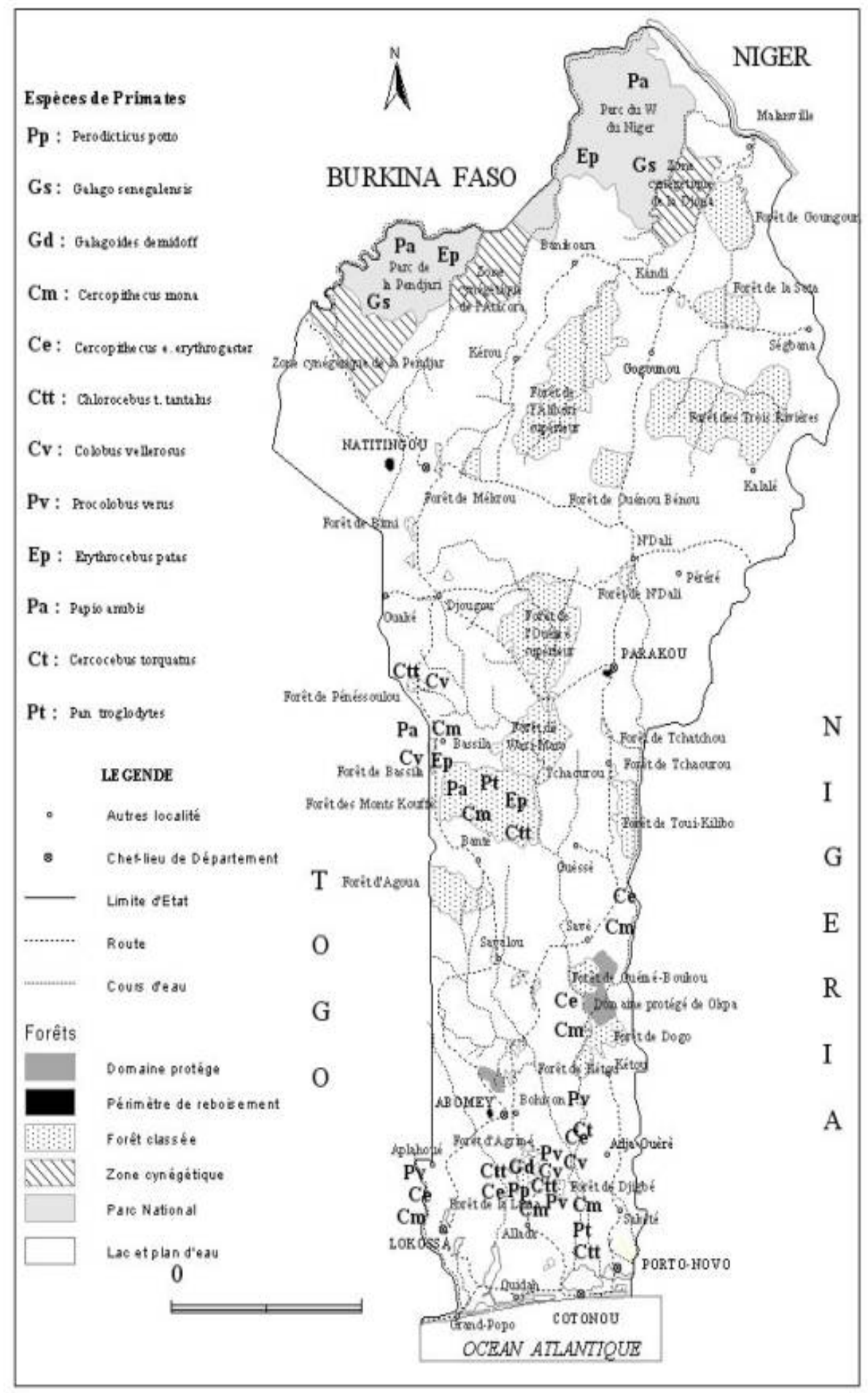

Figure 1: Carte de répartition des espèces de primates suivant les aires protégées au Bénin. 


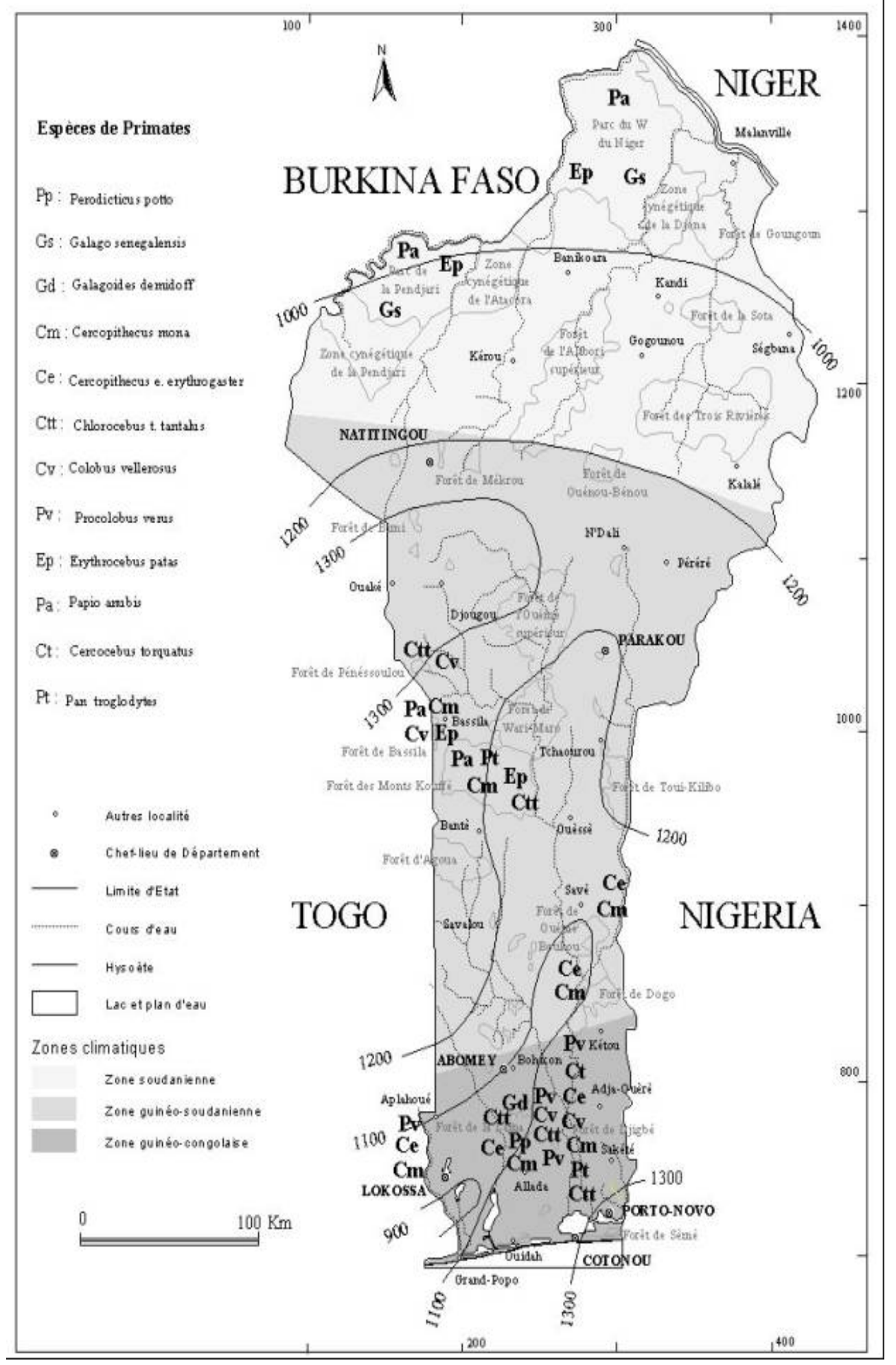

Figure 2: Carte de répartition des espèces de primates selon les zones climatiques du Bénin. 
Tableau 1: Diversité des espèces de primates du Bénin.

\begin{tabular}{|c|c|c|c|}
\hline $\begin{array}{l}\text { Espèces / Auteur / } \\
\text { Année / Famille }\end{array}$ & $\begin{array}{l}\text { Zones } \\
\text { climatiques }\end{array}$ & Ecosystèmes & Habitats/Sources/Dernière observation \\
\hline $\begin{array}{l}\text { Perodicticus potto } \\
\text { Müller, } 1766 \\
\text { Loridae }\end{array}$ & $\begin{array}{l}\text { Guinéo- } \\
\text { congolaise }\end{array}$ & $\begin{array}{l}\text { Forêt dense semi- } \\
\text { décidue }\end{array}$ & $\begin{array}{l}\text { FC Lama } \\
\text { Observations personnelles } ; 2000\end{array}$ \\
\hline $\begin{array}{l}\text { Galago senegalensis } \overline{\mathrm{E}} \text {. } \\
\text { Geoffroy, } 1796 \\
\text { Galagonidae }\end{array}$ & $\begin{array}{l}\text { Guinéo- } \\
\text { congolaise } \\
\text { Soudanienne } \\
\end{array}$ & $\begin{array}{l}\text { Forêt dense semi- } \\
\text { décidue }\end{array}$ & $\begin{array}{l}\text { PN W du Niger, PN Pendjari } \\
\text { Emrich et al.,1999; Kassa, 2001; Nobimè, 2002, } \\
2003\end{array}$ \\
\hline $\begin{array}{l}\text { Galagoides demidoff } \\
\text { Fischer, } 1806 \\
\text { Galagonidae }\end{array}$ & $\begin{array}{l}\text { Guinéo- } \\
\text { congolaise }\end{array}$ & $\begin{array}{l}\text { Forêt dense semi- } \\
\text { décidue }\end{array}$ & $\begin{array}{l}\text { FC Lama } \\
\text { Emrich et al., 1999; Kassa, 2001; Nobimè, 2002, } \\
2003\end{array}$ \\
\hline $\begin{array}{l}\text { Cercopithecus mona } \\
\text { Waterhouse, } 1838 \\
\text { Cercopithecidae }\end{array}$ & $\begin{array}{l}\text { Guinéo- } \\
\text { congolaise } \\
\text { Guinéo- } \\
\text { soudanienne }\end{array}$ & $\begin{array}{l}\text { Forêt dense semi- } \\
\text { décidue, forêt } \\
\text { galerie }\end{array}$ & $\begin{array}{l}\text { FC Lama, Vallées Ouémé et Mono, Bassila, } \\
\text { galerie Okpara } \\
\text { Emrich et al., 1999; Kassa, 2001; Nobimè, 2002; } \\
\text { Observations personnelles, 2007 }\end{array}$ \\
\hline $\begin{array}{l}\text { Cercopithecus e. } \\
\text { Erythrogaster } \\
\text { Gray, } 1866 \\
\text { Cercopithecidae }\end{array}$ & $\begin{array}{l}\text { Guinéo- } \\
\text { congolaise }\end{array}$ & $\begin{array}{l}\text { Forêt dense semi- } \\
\text { décidue, forêt } \\
\text { galerie }\end{array}$ & $\begin{array}{l}\text { FC Lama, vallée de l'Ouémé, vallée du Mono, } \\
\text { galerie Okpara } \\
\text { Emrich et al., 1999; Kassa, 2001; Nobimè, 2002; } \\
\text { Observations personnelles, 2007 }\end{array}$ \\
\hline $\begin{array}{l}\text { Chlorocebus t. tantalus } \\
\text { Linnaeus, } 1758 \\
\text { Cercopithecidae }\end{array}$ & $\begin{array}{l}\text { Guinéo- } \\
\text { congolaise, } \\
\text { Guinéo- } \\
\text { soudanienne } \\
\text { Soudanienne }\end{array}$ & $\begin{array}{l}\text { Forêt dense semi- } \\
\text { décidue, forêt } \\
\text { galerie }\end{array}$ & $\begin{array}{l}\text { FC Lama, vallée de l'Ouémé, FC Mt Kouffé } \\
\text { PN Pendjari, PN W du Niger, FC Pénéssoulou } \\
\text { Emrich et al., 1999; Kassa, 2001; Nobimè, 2002; } \\
\text { Sayer and Green, 1984; Kassa, 1998; Gaoué, } \\
\text { 2000; } \\
\text { Observations personnelles, } 2007\end{array}$ \\
\hline $\begin{array}{l}\text { Colobus vellerosus } \\
\text { Wesmael, } 1835 \\
\text { Cercopithecidae }\end{array}$ & $\begin{array}{l}\text { Guinéo- } \\
\text { congolaise } \\
\text { Guinéo- } \\
\text { soudanienne }\end{array}$ & $\begin{array}{l}\text { Forêt dense semi- } \\
\text { décidue, forêt } \\
\text { galerie }\end{array}$ & $\begin{array}{l}\text { FC Lama, forêt de Gnanhouizounmè, forêt } \\
\text { sacrée Kikélé, FC Pénéssoulou } \\
\text { Emrich et al., 1999; Kassa, 2001; Nobimè, 2002; } \\
\text { Djossou, 2003, } 2007\end{array}$ \\
\hline $\begin{array}{l}\text { Procolobus verus Van } \\
\text { Beneden, } 1838 \\
\text { Cercopithecidae }\end{array}$ & $\begin{array}{l}\text { Guinéo- } \\
\text { congolaise }\end{array}$ & $\begin{array}{l}\text { Forêt dense semi- } \\
\text { décidue, forêt } \\
\text { galerie }\end{array}$ & $\begin{array}{l}\text { FC Lama, Lokoli, vallée de l'Ouémé, vallée du } \\
\text { Mono, } \\
\text { Emrich et al., 1999; Kassa, 2001; Nobimè, 2002, } \\
2003\end{array}$ \\
\hline $\begin{array}{l}\text { Erythrocebus patas } \\
\text { Schreber, } 1775 \\
\text { Cercopithecidae }\end{array}$ & $\begin{array}{l}\text { Guinéo- } \\
\text { soudanienne } \\
\text { Soudanienne }\end{array}$ & $\begin{array}{l}\text { Savane, } \\
\text { claire }\end{array}$ & $\begin{array}{l}\text { Bassila, FC Mt Kouffé, PN Pendjari, PN W du } \\
\text { Niger, } \\
\text { Sayer and Green, 1984; Kassa, 1998; Gaoué, } \\
\text { 2000; } \\
\text { Observations personnelles, } 2007\end{array}$ \\
\hline $\begin{array}{l}\text { Papio anubis } \\
\text { Linnaeus, } 1758 \\
\text { Cercopithecidae }\end{array}$ & $\begin{array}{l}\text { Guinéo- } \\
\text { soudanienne } \\
\text { Soudanienne }\end{array}$ & $\begin{array}{l}\text { Savane, } \\
\text { claire }\end{array}$ & $\begin{array}{l}\text { Bassila, FC Mt Kouffé, PN Pendjari, PN W du } \\
\text { Niger } \\
\text { Sayer and Green, 1984; Kassa, 1998; Gaoué, } \\
\text { 2000; } \\
\text { Observations personnelles, } 2007\end{array}$ \\
\hline $\begin{array}{l}\text { Cercocebus torquatus } \\
\text { Kerr, } 1792 \\
\text { Cercopithecidae }\end{array}$ & $\begin{array}{l}\text { Guinéo- } \\
\text { congolaise }\end{array}$ & $\begin{array}{l}\text { Forêt dense semi- } \\
\text { décidue }\end{array}$ & $\begin{array}{l}\text { FC Lama, Lokoli } \\
\text { Enquêtes, vers } 2000\end{array}$ \\
\hline $\begin{array}{l}\text { Pan troglodytes? } \\
\text { Hominidae }\end{array}$ & $\begin{array}{l}\text { Guinéo- } \\
\text { congolaise } \\
\text { Guinéo- } \\
\text { soudanienne } \\
\end{array}$ & $\begin{array}{l}\text { Forêt dense semi- } \\
\text { décidue }\end{array}$ & $\begin{array}{l}\text { FC Mt Kouffé, Forêt de Gnanhouizounmè } \\
\text { Heymans et Petit, 1985; enquêtes, mémoire de } \\
\text { chasseur }\end{array}$ \\
\hline
\end{tabular}

La zone guinéo-soudanienne comporte des espèces comme le tantale, le patas, le babouin, la mone et le colobe de Geoffroy. Si le patas, le tantale et le babouin sont les espèces les plus communes dans cette zone, la mone et le colobe de Geoffroy sont, eux- autres, confinées aux galeries forestières et autres îlots de forêts denses.

Enfin dans la zone soudanienne, on note la présence des espèces comme le babouin, le patas, le tantale et le galago de Sénégal. Ce sont des espèces communes aux 
milieux savanicoles. La république du Bénin, malgré son appartenance au "sillon dahoméen", dispose de ressources appréciables en primates.

\section{Quelques aspects ethnozoologiques des primates}

L'être humain apprécie les ressources biologiques de manières différentes : spirituellement, économiquement, esthétiquement, culturellement et scientifiquement. Pour les communautés locales, les ressources biologiques représentent la source principale de vie, de médicaments et de valeurs spirituelles.

Chez la plupart des groupes socioculturels du Bénin, les primates représentent les jumeaux. Ainsi ces animaux sont vénérés et constituent des tabous pour des personnes ayant un lien ou une parenté avec les jumeaux. Ces derniers ne chassent ni ne consomment la viande de singe. Ils ne doivent faire aucun mal aux singes. Mais cette tradition, depuis quelques années, n'a pu résister à la vague des nouvelles religions chrétiennes pour lesquelles ces valeurs traditionnelles de conservation de la faune sont foulées au pied.

Les organes de certaines espèces de primates sont utilisés comme ingrédients dans la préparation de remèdes et autres produits médico-magiques. Le tableau 2 donne quelques exemples de valeurs ethnozoologiques des primates chez les communautés "Fon", "Nagot" et "Berba" du Bénin.

\section{DISCUSSION}

Les précipitations constituent le principal facteur déterminant pour les habitats des mammifères (Sayer \& Green, 1984). La forêt classée de la Lama abrite les espèces comme la mone, le cercopithèque à ventre rouge, le tantale, le colobe de Geoffroy, le colobe de Van Beneden, des galagos et le potto de Bosman (Kafichoni, 1987; Kassa, 2001; Nobimè, 2002; Nobimè et Sinsin, 2003 ; Djossou, 2003 ; Campbell et al., 2008). La vallée du fleuve Ouémé regorge d'îlots forestiers qui abritent quelques espèces de primates. Sinsin et al. (2000) ont distingué les espèces comme la mone, le cercopithèque à ventre rouge, le colobe de Van Beneden et le tantale. La dégradation des ressources naturelles dans ce milieu fait que ce sont les reliques de forêt et les jachères anciennes qui abritent la faune sauvage. Dans la forêt marécageuse de Lokoli, Sinsin et Assogbadjo (2002) ont noté la présence de la mone, du tantale, du colobe de Geoffroy, du cercopithèque à ventre rouge. Malgré la pression sur les ressources naturelles dans cette zone guinéo-congolaise, les divers taxa ont su s'adapter à la modification de leurs habitats originels, sauf le mangabey pour lequel aucun individu n'est encore observé.

Tableau 2: Quelques valeurs ethnozoologiques des primates.

\begin{tabular}{|c|c|c|}
\hline Espèces de primates & $\begin{array}{l}\text { Organes } \\
\text { utilisés }\end{array}$ & Remèdes/produits médico-magiques \\
\hline $\begin{array}{l}\text { Potto de Bosman } \\
\text { Perodicticus potto }\end{array}$ & Crâne & Remède pour soigner la fontanelle chez l'enfant \\
\hline $\begin{array}{l}\text { Colobe de Geoffroy } \\
\text { Colobus vellerosus }\end{array}$ & Crâne & $\begin{array}{l}\text { Produits médico-magiques pour créer la division au sein } \\
\text { d'un groupe }\end{array}$ \\
\hline $\begin{array}{l}\text { Colobe de Geoffroy } \\
\text { Colobus vellerosus }\end{array}$ & $\begin{array}{l}\text { Toute la } \\
\text { dépouille }\end{array}$ & Produits médico-magiques pour se rendre invisible \\
\hline Babouin Papio anubis & Crâne & $\begin{array}{l}\text { Produits médico-magiques pour le "Koudio" à la fin de } \\
\text { l'année }\end{array}$ \\
\hline Patas Erythrocebus patas & Langue & $\begin{array}{l}\text { Le singe est tué et sa langue est récupérée et séchée. Elle } \\
\text { est conservée, et en cas de hoquet, il faut la tremper dans } \\
\text { un peu d'eau fraîche pendant quelques minutes. L'eau est } \\
\text { récupérée pour boisson et calme le hoquet. }\end{array}$ \\
\hline $\begin{array}{l}\text { Cercopithèque à ventre } \\
\text { rouge Cercopithecus } e . \\
\text { erythrogaster }\end{array}$ & La dépouille & Produits médico-magiques pour traiter la démence, la folie \\
\hline Cercopithèques & Crâne & $\begin{array}{l}\text { Crânes utilisés pour servir aux bébés jumeaux leur } \\
\text { première boisson d'eau, ainsi le singe ne sera plus pour eux } \\
\text { un tabou }\end{array}$ \\
\hline
\end{tabular}


La région des Monts Kouffé a un climat soudano-guinéen caractérisé par une seule saison sèche (novembre à avril). Heymans et Petit (1985) signalent la présence de primates tels que la mone, le tantale, le patas et le babouin. Pour ces mêmes auteurs, la forêt classée des Monts Kouffé renferme peut-être des chimpanzés. Les ressources fauniques de la région de Bassila comportent les espèces de primates suivantes : le babouin, le patas, le vervet, le colobe magistrat et le colobe olive (Sinsin, 1996). Dans la forêt classée de Toui-Kilibo, Sinsin et al. (2002) ont observé deux espèces de primates, le patas et le vervet.

Dans la zone soudanienne, Sinsin (1998) signale le babouin, le patas et le vervet dans les forêts classées des Trois Rivières, de Ouenou-Benou et de l'Alibori. Les mêmes espèces de primates sont observées dans les forêts classées de Sota et Goungoun (Sinsin et al., 1997), dans le Parc National W (Kassa, 1998), dans la Zone Cynégétique du Parc National de la Pendjari (Sinsin et al., 1996 ; Gaoué, 2000). Dans les parcs nationaux W et Pendjari du Bénin, les espèces de primates comme le galago (Galago senegalensis), le vervet, le patas et le babouin sont observées (Kassa, 1998 ; Gaoué, 2000).

Le pétauriste Cercopithecus petaurista et le pain à cacheter $C$. nictitans cités comme anciens habitants du "Dahomey Gap" ont localement disparu (Emrich et al., 1999). Pour Sinsin (1995), ceci montre qu'il n'est pas exclu que les espèces disparues de la forêt classée de la Lama soient éteintes tout au moins à l'échelle du pays et même à l'échelle mondiale si c'est une espèce endémique du Bénin. Le mangabey et le chimpanzé auraient certainement disparu car aucune observation n'a été faite actuellement. Mais les trophées $\mathrm{du}$ premier existent encore auprès des communautés locales (photo 1). Dans la forêt de Gnanhouizounmè située dans la même dépression que celle de la Lama, les chasseurs reconnaissent l'existence du chimpanzé dans un passé récent.

La déforestation constitue la principale menace qui pèse sur les primates du Bénin. Dans les terroirs villageois, l'agriculture itinérante engendre la perte de la végétation originelle, et par conséquent de l'habitat des singes. Dans les aires protégées, c'est surtout la chasse illégale qui menace la survie des espèces de primates.

La liste des valeurs ethnozoologiques des primates chez les communautés "Fon", "Nagot" et "Berba" dans la présente étude n'est pas limitée. Il s'agit ici d'une ébauche d'ethnozoologie sur les primates du Bénin. La collecte des données auprès de la majorité des groupes socio-culturels du pays permettra de mieux cerner l'importance des primates dans l'ethnozoologie au Bénin.

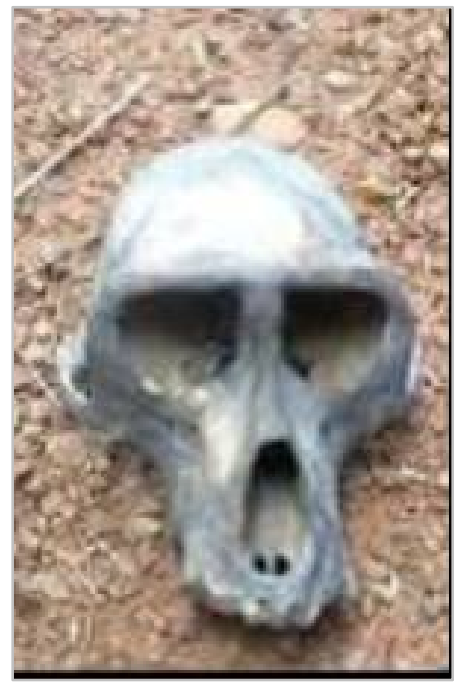

Photo 1: Crâne du mangabey

\section{Conclusion}

La république du Bénin, malgré sa place dans le Dahomey-Gap, abrite de nos jours 10 espèces et sous-espèces de primates confirmées et 1 espèce qui n'est pas encore confirmée. Les galeries forestières ont réussi à conserver une part importante de la diversité des primates forestiers. Ainsi le cercopithèque à ventre rouge et la mone se retrouvent dans les galeries forestières de l'Ouémé, de l'Opkara et du Mono.

Une prospection rigoureuse des différents écosystèmes du pays permettra de mieux renseigner sur la diversité en primates. Des espèces comme le mangabey, le pétauriste et le chimpanzé peuvent avoir des survivants qui ont échappé à l'extinction. La communauté internationale est alors interpellée pour soutenir la recherche scientifique sur l'identification des primates existant dans le sillon dahoméen. Des institutions de conservation des primates 
écartent le Bénin des pays à forte diversité de primates forestiers, mais un travail de prospection pourrait vraisemblablement faire évoluer ce positionnement. Avec huit espèces de primates forestiers dont une sous-espèce endémique, le cercopithèque à ventre rouge, nous pensons que le Bénin mérite d'être considéré comme les autres pays forestiers de l'Afrique de l'Ouest. Même si le DahomeyGap forme une discontinuité faunistique significative pour les espèces de forêt, il n'est pas pour autant vide en primates forestiers.

\section{REMERCIEMENTS}

Toutes nos gratitudes aux populations des différentes localités qui nous ont accueillis sur le terrain, et toutes les personnes qui nous ont aidés dans la collecte des informations. Nos remerciements à tous les volontaires de Planète Urgence qui sont arrivés au Bénin pour les missions de conservation des ressources naturelles.

\section{REFERENCES BIBLIOGRAPHIQUES}

Campbell G, Teichroeb J, Paterson JD. 2008. Distribution of diurnal primates species in Togo and Benin. Folia Primatologica 79(1): $15-30$

Djossou GS. 2003. Détermination de l'aire de répartition de la population de colobe magistrat Colobus vellerosus et statuts de conservation au Bénin. DESS, FSA/Université d'Abomey-Calavi, Bénin, p.97.

Emrich A, Mühlerberg H, Steirhauer-Burkart, Sturm HJ. 1999. Rapport de synthèse. Evaluation écologique intégrée de la forêt naturelle de la Lama en République du Bénin. Elaboré pour le compte de "Promotion de l'économie forestière et du bois" ONAB, KFW, GTZ, ECO Gesellschaft für socialökologische Programmberatung, Cotonou, Bénin, 74p.

Gaoue OG. 2000. Facteurs déterminants pour le zonage de la zone cynégétique de la Pendjari comme base de gestion intégrée. Mém. d'Ir. Ag. FSA/UNB AbomeyCalavi, Bénin, p. 106.

Heymans J-C, Petit JM. 1985. Etude et aménagement de la forêt classée des Monts Kouffé (Rép. du Bénin). Tropicultura . 3(3): 88-92.

Jenik J. 1994. The Dahomey-Gap: an important issue in African phytogeography. Mémoire de la Société Biogéographique (3ème série) IV: 125133.

Kafichoni B. 1987. Etude écoéthologique d'un primate: Cercopithecus aethiops tantalus dans la zone sud de la Forêt Classée de la Lama. CPU/UNB: Abomey-Calavi, Bénin; p. 67.

Kassa DB. 1998. Problématique de l'aménagement écologique pour la conservation des ressources fauniques dans le Parc National du W: Périmètre de Kérémou au Nord-Bénin. Mém. d'Ir. Ag. FSA/UNB Abomey-Calavi, Bénin, p. 168.

Kassa DB. 2001. Techniques de dénombrement et facteurs déterminant la modélisation de la dynamique de la faune sauvage dans la forêt dense semi-décidue de la Lama. DESS, FSA/UNB, AbomeyCalavi, Bénin, p 92.

Le Gall Ph, Goergen G, Neuenschwander P. 2002. Les insectes et le sillon dahoméen: fragmentation et refuges forestiers. Biosystema, Systématique et biogéographie, 20: 73-80.

Natta AK. 2003. Ecological assessment of riparian forests in Benin: Phytodiversity, phytosociology and spatial distribution of tree species. Ph.D. Thesis, Wageningen University, Pays-Bas, p. 215.

Nobimè G. 2002. Collecte de données de base pour la protection du singe à ventre rouge Cercopithecus erythrogaster erythrogaster dans la forêt classée de la Lama au Bénin. DEA, EDP/GE/FLASH, Université d'Abomey-Calavi, Bénin, p. 75.

Nobimè G, Sinsin B. 2003. Les stratégies de survie du singe à ventre rouge (Cercopithecus erythrogaster erythrogaster) dans la forêt classée de la Lama au Bénin. Biogeographica 79(4): 153-166.

Oates JF. 1988. The distribution of Cercopithecus monkeys in West African forests. In A Primate Radiation: Evolutionary Biology of the African Guenons, Gautier-Hion A, Bourlière F, Gautier J-P, Kingdon J (eds). Cambridge University Press; 79-103.

Refisch J. 1998. Singe et Autres Mammifères. Evaluation Ecologique Intégrée de la Forêt Naturelle de la Lama en République du Bénin. ECO, ONAB, KfW/GTZ: Cotonou, Bénin; 27. 
Sayer JA, Green AA. 1984. The distribution and status of large mammals in Benin. Mammal. Rev. 14(1): 37-50.

Sinsin B. 1996. Les Ressources Fauniques et leur Conservation dans la Région de Bassila. Projet Bois de Feu/DFRN/MDR: Cotonou, Bénin; 23.

Sinsin B. 1998. Etude de la faune et de son habitat dans les forêts classées des trois rivières, de Ouénou-Bénou et de l'Alibori Supérieur. Rapport de Mission, Ambassade Royale des Pays-Bas, Cotonou, Bénin, p. 47.

Sinsin B, Daouda I-H, Ahokpe E, Faaki V, Agonyissa D, Houinato M, Toko I, Oumorou M, Kindomihou V, Yaoitcha J. 1996. Dénombrement de la faune dans la zone cynégétique de la Pendjari. Rapport de Mission, PGRN, Cotonou, Bénin.

Sinsin B, Daouda I-H, Ahokpè E, Tehou A, Coubeou P, Toko I, Koto Mity B. 1997. Programme de conservation des ressources fauniques dans les forêts classées de Goungoun et de la Sota. Document de synthèse. Rapport de mission, DFRN-UNSO, Cotonou, Bénin.
Sinsin B, Daouda I-H, Ahokpè E. 1998. Abondance et évolution des populations de mammifères des formations boisées de la région des monts Kouffé au Bénin. Cahiers d'éthologie, 18(2): 261-281.

Sinsin B, Assogbadjo EA. 2002. Diversité, structure et comportement des primates de la forêt marécageuse de Lokoli au Bénin. Biogeographica 78(4): 129-140.

Sinsin B, Tehou AC, Nobimè G, Tchibozo $\mathrm{S}$. 2000a. Répartition et Abondance du Singe à Ventre Rouge Cercopithecus erythrogaster dans les Régions de la Lama et d'Adjohoun (Bas-Bénin). LEA/FSA/UNB: Cotonou, Bénin; 33.

Sinsin B, Saidou A, Daouda IH, Tehou A, Nobimè G. 2000b. Dénombrement de la faune sauvage dans le complexe Pendjari. Rapport technique de mission. CENAGREF, Projet Pendjari, GTZ, Cotonou, Bénin.

Sinsin $\mathrm{B}$, Nobimè $\mathrm{G}$, Tehou $\mathrm{A}$, Bekhuis $\mathrm{P}$, Tchibozo S. 2002. Past and present distribution of the red-bellied monkey Cercopithecus erythrogaster erythrogaster in Benin. Folia Primatologica, 73: 116-123. 